\title{
Lung volume irradiation procedures in patients with pneumonia during COVID-19 infection - physical aspects of treatment planning and dosimetry
}

\author{
Krzysztof Ślosarek1, Adam Gądek , Łukasz Sroka' , Łukasz Dolla1, Andrzej Biały', Michał Radwan', \\ Dawid Bodusz ${ }^{2}$, Michał Nachlik², Tomasz Rutkowski³ , Jerzy Jaroszewicz ${ }^{4}$
}
'Radiotherapy Planning Department, Maria Sklodowska-Curie National Research Institute of Oncology, Gliwice Branch, Gliwice, Poland ${ }^{2}$ Radiotherapy Department, Maria Sklodowska-Curie National Research Institute of Oncology, Gliwice Branch, Gliwice, Poland 31-st Radiotherapy Clinic, Maria Sklodowska-Curie National Research Institute of Oncology, Gliwice Branch, Gliwice, Poland ${ }^{4}$ Observation and Infection and Hepatology Department of Specialist Hospital No. 1 in Bytom, Bytom, Poland

Introduction. The paper presents an original, used in our Institute, method of lung volume irradiation in patients with pneumonia during COVID-19 infection.

Material and methods. Procedures such as the simulation of treatment and radiotherapy are performed in a treatment room. Real time radiation treatment planning is realized as 2D planning (Irreg Planning VMS) in a separate room, and the $3 \mathrm{D}$ (eclipse VMS) dose distribution is calculated after the treatment. During radiation exposure, a fluence map is measured. Results. A method of irradiating the lungs of patients with COVID-19 was developed, which allows to shorten the time the patient is on the treatment table and minimize contacts between the patient and staff.

Conclusions. The presented procedure made it possible to minimize the time of patient's stay in the radiotherapy department and at the same time, it retains all the required quality assurance procedures in radiotherapy treatment.

Key words: COVID-19, cone beam computed tomography, 2D and 3D treatment planning, a fluence map

\section{Introduction}

The use of ionizing radiation in the treatment of pneumonia and other inflammatory conditions has a long tradition [1-3]. In contemporary literature [4-7], information can be found on the use of low-dose rate radiotherapy in the treatment of inflammation, including pneumonia. The current pandemic situation is characterized by the dramatic course of pneumonia among certain COVID-19 patients and the relatively high percentage of deaths due to lung failure in this group. Therefore, the question should be raised as to whether low-dose radiotherapy can become a treatment method that gives a chance to improve the quality of life and reduce the risk of death for such patients. Such a treatment protocol should determine a radiation dose per lung volume area [8-10] and be positively assessed by the Bioethics Committee.

\section{Objective}

The aim of this paper is to present a unique and proprietary procedure of lung radiotherapy in pneumonia patients during

\section{How to cite:}

Ślosarek K, Gądek A, Sroka Ł, Dolla Ł, Biały A, Radwan M, Bodusz D, Nachlik M, Rutkowski T, Jaroszewicz J. Lung volume irradiation procedures in patients with pneumonia during COVID-19 infection - physical aspects of treatment planning and dosimetry. NOWOTWORY J Oncol 2021;71:238-242. 
the course of COVID-19 infection with particular emphasis on the physical aspects of treatment planning and dosimetry. The duration of the procedure (planning and treatment) should be as short as possible.

1. The place of the procedure should be limited to a minimum number of rooms. The irradiation session should be image guided.

2. The target area refers to the irradiated volume, namely the patient's lungs. The only organ shielded is the spinal canal.

3. The dose distribution should be presented in 3D.

Based on pre-clinical and clinical studies [8-10], a dose in the range: $0.5-1.5 \mathrm{~Gy}$ is considered to have anti-inflammatory properties. Given this, the mean dose in this procedure was set at $1 \mathrm{~Gy}$ in whole lungs volume.

\section{Material and methods}

Considering the epidemic risk, it was assumed that the patients should be admitted to the Radiotherapy Department through a separate entrance, so as not to cross communication routes dedicated to oncological patients and medical staff. Additionally, both radiotherapy preparation and treatment should take place in a combined complex of rooms isolated from other patients and staff.

One of the TrueBeam Varian Medical Systems (VMS) accelerators was installed near the service entrance to the RT Department. It is equipped with an OBI (On-Board Imager): CBCT and kV/MV imaging. Software Irreg Planning, is an integrated part of the Aria System (16.1.0) VMS, which makes it possible to calculate the treatment time ( $M U$ - monitor units) based on the size of the irradiation field (beam), source skin distance (SSD) and the depth of the planning dose definition. Therefore, the MUs is calculated for a density of $1 \mathrm{~g} / \mathrm{cm}^{3}$. When this software (Irreg Planning) is used in the irradiation of the chest volume, the calculated treatment time is overstated. This software (2D planning) is used for clinical purposes during simulation in cases where it is particularly important to shorten the preparation time of patients to radiotherapy e.g., analgesic therapy. In the case of a single irradiation session, a CT scan is not required to be performed.

It was assumed that the calculated dose distribution would be three-dimensional, in order to assess the statistical dose in the lungs and critical organs: the heart and the spinal cord. The TrueBeam accelerator is equipped with both $\mathrm{kV}$ and MV (on board imager - OBI) imaging systems; $C B C T$ is a routine procedure to verify the patient's position before a therapeutic session. Until now, this study has not been used to plan dose distributions. In this case, however, in order to shorten the overall duration of the treatment procedure, CBCT was used to calculate the dose distribution.

With regard to the clear reduction of the procedure time, it was decided that treatment would be carried out using the TrueBeam VMS. The following procedure stages were developed (fig. 1):

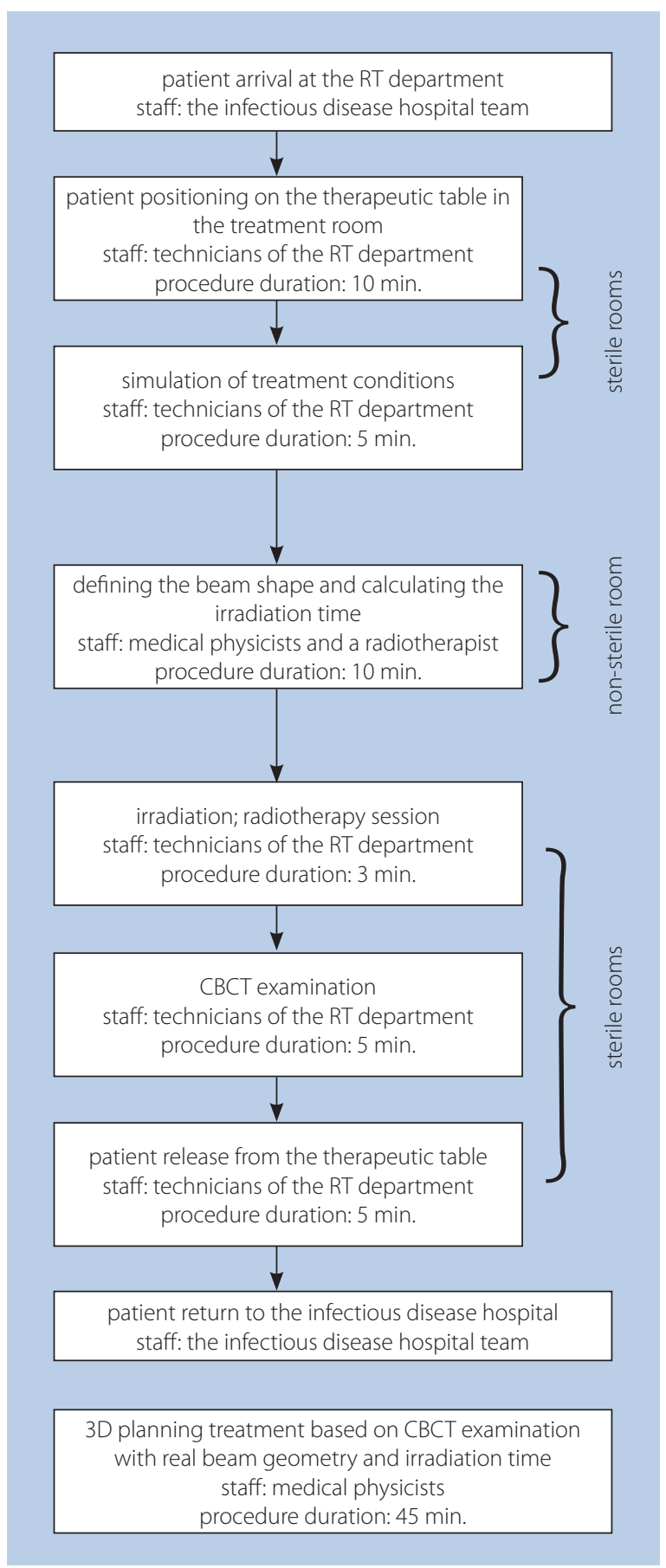

Figure 1. A schematic diagram of patient irradiation procedures. The time the patient stays in the therapeutic position on the table is 30 minutes. The total stay in the RT Department is limited to 45 minutes

1. The patient is placed in a therapeutic position on the table of the therapeutic apparatus.

2. With the use of kV (X-ray) imaging, the irradiation conditions are simulated by defining the maximum dimensions of the therapeutic beam. The AP (anterior-posterior) dimension is defined in the transverse largest dimension at lung level. Based on this measurement, the depth (half AP dimension) of prescribed dose is determined. 
3. Based on the simulation image (one, at an angle of $0^{\circ}$ ) in the Irreg Planning software, MLC is introduced - limiting the irradiation area to the contour of the lungs with the spinal canal being shielded (the MLC collimator should be set at $90^{\circ}$ ). After copying the field shape defined in this way to the opposite beam, at an angle of $180^{\circ}$, the software adjusts the position of the collimator leaves to the new irradiation angle. The irradiation time is calculated.

4. The treatment planning (2D) process is completed by entering the beam parameters (i.e. MU, MLC, table, gantry, collimator position) into the patient management system (ARIA).

5. The patient is irradiated and the fluence map is measured by EPID., and at the end of exposure, a CBCT scan is performed.

6. The patient completes the treatment and is taken back to their hospital base.

7. Dose distribution calculations (3D) are performed for the implemented radiation conditions, i.e., the shape of the radiation beam and exposure time. This stage is designed to accurately determine the dose received by the patient in the volume of each defined area.

\section{Dosimetric preparation}

The Irreg Planning software does not consider the heterogeneity of the irradiated medium, and thus the actual radiation dose in this volume can be expected to be higher, since the lung density is less than the water density $-1 \mathrm{~g} / \mathrm{cm}^{3}$. That is why it should be verified by calculations based on CT scans. The precise calculation of dose distributions requires the introduction of the calibration curve of the device used for imaging into the treatment planning system (TPS-Eclipse VMS 16.01.03). In this case, it was the OBI-CBCT device of the TrueBeam accelerator. Unfortunately, this proved to be difficult to implement, and thus the differences were checked between the dose distribution calculations made with $C T$ scans for routine treatment planning and $C B C T$, using the reference calibration curve (fig. 2). For this purpose, phan- toms were utilized to calibrate the CT scanner (CIRS-Norfolk, Virginia, USA). Due to the slight differences between the calculations: maximum dose, the examination performed with a calibrated CT scanner - $2.173 \mathrm{~Gy}$, and for CBCT, with a reference calibration curve $-2.176 \mathrm{~Gy}$, it was found that it was possible to perform dose calculations for the patient using the CBCT scanner.

Before the first treatment procedure, all the steps described above were taught to the staff (RT technicians, medical physicists). It was established that in order to optimize the duration of the treatment, the patient's irradiation procedure should be carried out first; only at the end of exposure should a conical CT scan be performed. The width of the area that can be examined during one CT scan is $20 \mathrm{~cm}$. Since the examined lung volume is a much larger area, two CBCT scans need to be performed so that they can be later combined to determine the entire lung volume needed for calculation. This requires changing the centering points, which involves moving the therapy table. The CBCT scan is performed in the SAD technique and the patient is treated in the SSD technique. This requires changing the position of the therapy table.

\section{The first patient}

The patient was admitted to the RT Department on December $15^{\text {th }}, 2020$ at 5:12 pm. A 50-year-old woman with COVID-19 (based on a real time polymerase chain reaction of SARS-CoV-2 RNA) has been admitted to the hospital with symptoms and radiographic pneumonic consolidations of COVID-related pneumonia. 96 hours after admission, an RT procedure was carried out. Oxygen saturation level $\left[\mathrm{SpO}_{2}\right]$ with $\mathrm{O}_{2}$ supplementation via facial mask with reservoir bag was $88 \%$ prior to RT.

The first procedure involved placing the patient on a therapeutic table in the treatment position - on their back with their hands along their body and head supported (part of the AiO system - ORFIT company). No immobilization systems were used. A simulation image was produced using the MV beam. Then, the shape of the irradiation field was defined by determining the lung area by placing the collimator leaves

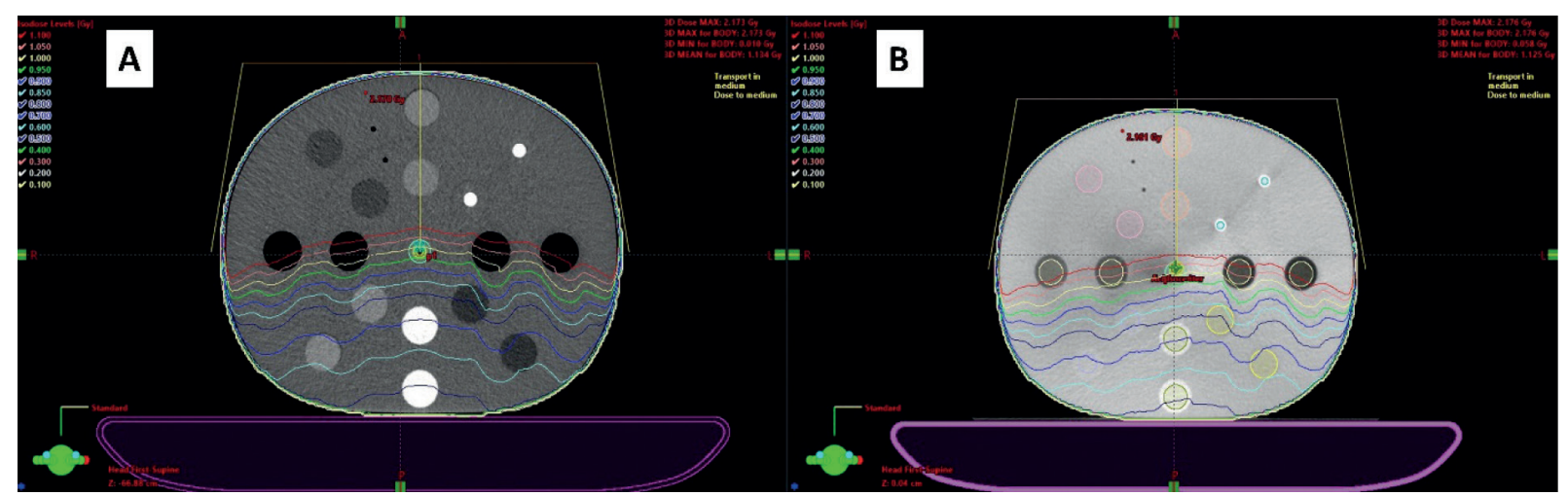

Figure 2. A comparison of phantom dose distributions with different densities. A. CT scans obtained by a CT scanner dedicated for treatment planning (AS-Siemens), a calibration curve introduced into the treatment planning system; B. scans obtained by a therapeutic device, with CBCT. Dose distribution calculations were made with the use of the treatment planning system Eclipse v 16.1.0 by Varian Medical Systems based on the reference calibration curve 


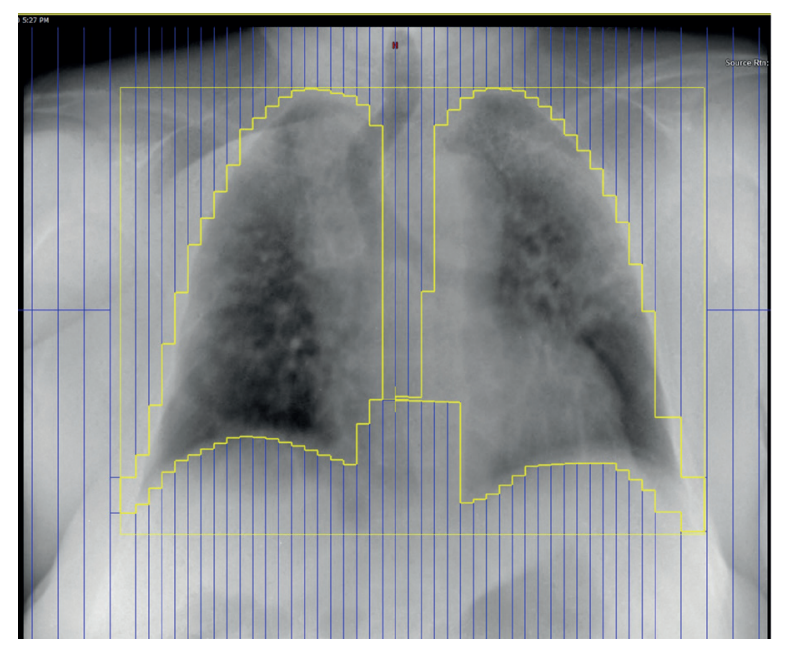

Figure 3. A simulation image with a prepared beam shape - MLC, beam entry at an angle of $0^{\circ}$. One should remember to set the collimator angle to $90^{\circ}$; to use the collimator leaves to shield the spinal canal (the direction of the collimator leaves' movement). The position of the collimator leaves is copied to the opposite beam at an angle of $180^{\circ}$

on the image obtained in the MV examination. Finally, the irradiation field determined at an angle of $0^{\circ}$ was copied to the opposite beam at an angle of $180^{\circ}$ by adjusting the shape of MLC (fig. 3).

The next stage involved calculating the irradiation time, for a dose of $1 \mathrm{~Gy}$, at a depth equal to $1 / 2$ of the AP (anterior posterior; in this case, it was $13.7 \mathrm{~cm}$ ). The $X$-ray beam of $6 \mathrm{MV}$ was applied and the SSD technique $(100 \mathrm{~cm})$ was used. The calculations were performed using the Irreg Planning 16.1.0 software by Varian Medical Systems [11], which is dedicated to calculations in 2D planning.

The patient was irradiated and a CBCT scan was performed at the end of the exposure. Due to the maximum volume that can be obtained in one CBCT rotation, two scans had to be performed, moving them relative to each other to cover the entire lungs.

During radiation exposure, a fluence map was measured on the extended EPID (Electronic Portal Imaging Devices) using the integrated dose option of the Varian Medical Systems'ARIA 16.1.0 software for both $0^{\circ}$ and $180^{\circ}$ radiation beams. The patient left the Radiotherapy Department at 5:50 pm. The whole described procedure lasted 38 minutes in total. However, the patient was on the therapeutic table, in the treatment position for only 20 minutes.

The 3D dose distribution planning, based on $C B C T$, is performed without the patient's presence in the RT Department. The dose distribution (3D) is calculated in the Eclipse v 16.1.0 VMS planning system, for irradiation time and beam geometry predefined in the Irreg Planning - ARIA VMS software (fig. 4). It is worth emphasizing at this point once again that this procedure is performed without the presence of the patient in the RT Department. It aims to precisely determine the dose received by the patient as the dosage calculation algorithm (Acuros 16.1.0-VMS) considers the actual tissue density obtained from CBCT.

The calculated dose distributions (Acuros algorithm 16.1.0-VMS), considering heterogeneity in density, indicate that the patient received an average dose of $1.091 \mathrm{~Gy}$ in the lungs. The average dose in the lungs was found to be higher than assumed, since the time calculated in the Irreg Planning option was for a medium with a density of $1 \mathrm{~g} / \mathrm{cm}^{3}$, while, in fact, lung tissue has a lower density. This makes the actual doses slightly higher. The modal dose in the spinal cord was 0.016 Gy. These differences do not exceed the therapeutic assumptions and significantly shorten the duration of the entire treatment procedure.

24 hours after the RT procedure, an increase in $\mathrm{SatO}_{2}$ was noticed and continued over the next days. 24 hours after RT, there was also significant decrease in CRP, IL6 and ferritin

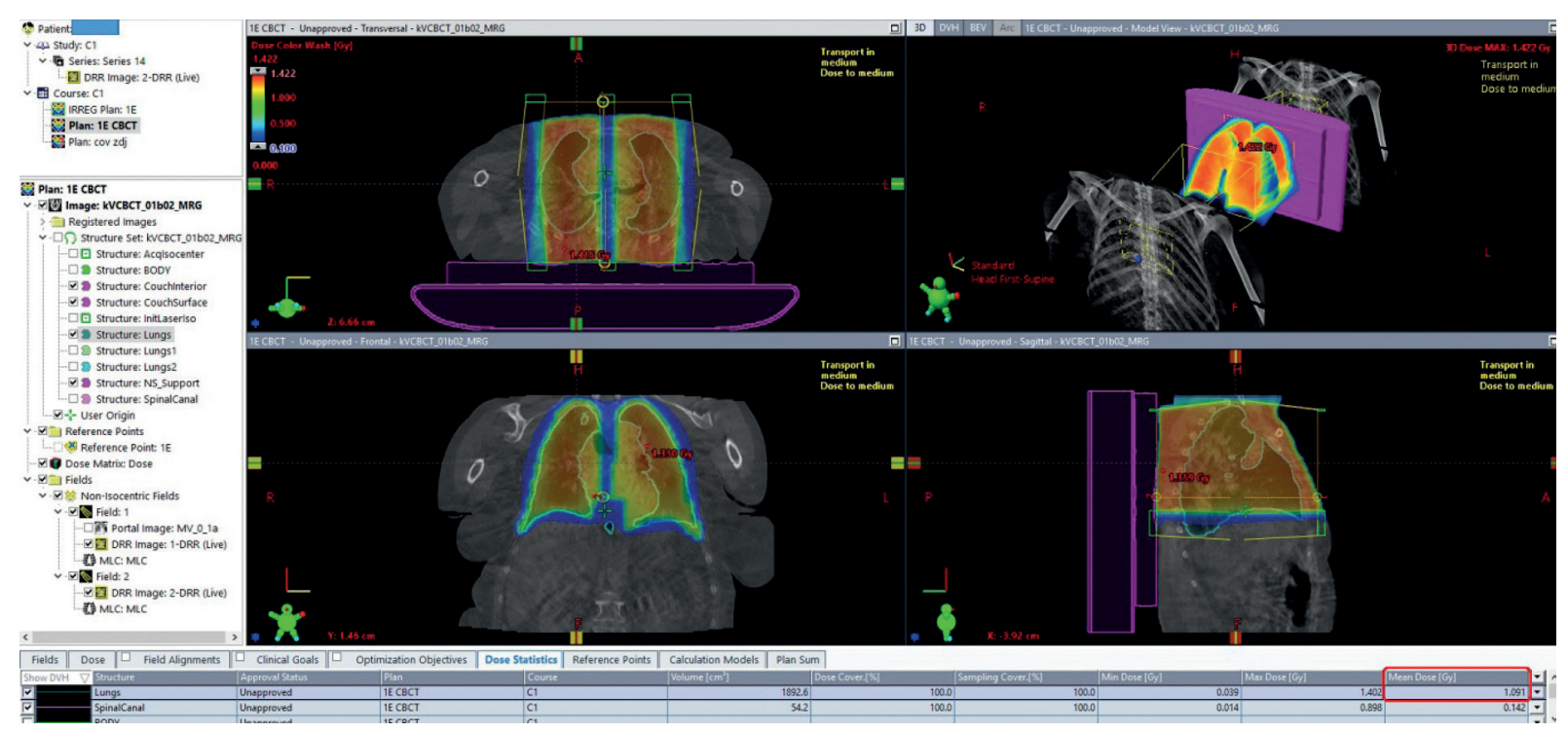

Figure 4. Dose distributions calculated for beam geometry: shapes and the number of monitoring units previously calculated in the Irreg Planning-VMS software 16.1.0 
Table I. The change of biochemical parameters in the selected days after RT

\begin{tabular}{|lccccc|}
\hline Marker/day & 0 & 1 & 5 & 7 & 14 \\
\hline Sat & $88 \%$ & $90 \%$ & $94 \%$ & $97 \%$ & $92 \%$ \\
\hline CRP & 166.73 & 7.68 & 2.47 & 1.58 & 1.58 \\
\hline Ferrytyna & 2453 & 685.52 & 783.95 & 601.95 & 556.76 \\
\hline IL6 & 23.38 & 2.04 & 3.06 & 2.19 & \\
\hline
\end{tabular}

Sat - oxygen saturation; CRP - C-reactive protein; IL6 - interleukin 6

level (tab. I). Clinically, improvement started 24 hours after RT and continued to the state that the patient was discharged from the hospital 14 days later with their overall status being very good.

\section{Conclusions}

The presented procedure of lung irradiation in patients with pneumonia during the course of COVID-19 infection made it possible to minimize the patient's stay in the Radiotherapy Department. At the same time, it ensures all the required radiotherapy treatment quality assurance procedures are adhered to. The dose received by the patient was consistent with the therapeutic requirements. The irradiation process was controlled from both a dosimetric (a fluence map) and an imaging (real-time MV imaging) perspective. The procedure also involved the calculation of three-dimensional dose distribution, which allows for the presentation of full statistics of dose distribution to the critical organs (spinal cord, heart).

\section{Conflict of interest: none declared}

\section{Adam Gądek}

Maria Sklodowska-Curie National Research Institute of Oncology

Gliwice Branch

Radiotherapy Planning Department

ul. Wybrzeże Armii Krajowej 15

44-102 Gliwice, Poland

e-mail:adam.gadek@io.gliwice.pl
Received: 1 Apr 2021

Accepted: 23 May 2021

\section{References}

1. ECalabrese EJ, Dhawan G. How Radiotherapy Was Historically Used To Treat Pneumonia: Could It Be Useful today ? Yale Journal Of Biology And medicine. 2013; 86: 555-570i.

2. Park SH, Lee J. Radiotherapy, a New Treatment Option for Non-malignant Disorders: Radiobiological Mechanisms, Clinical Applications, and Radiation Risk. Journal of Rheumatic Diseases. 2017; 24(2): 74, doi: 10.4078/jrd.2017.24.2.74

3. Musser JH. A study of metabolism in leukaemia, under the influence of the x-ray. TraAm Physician. 1905; 20: 294-323.

4. Arenas M, Gil F, Gironella M, et al. Anti-inflammatory effects of low-dose radiotherapy in an experimental model of systemic inflammation in mice. Int J Radiat Oncol Biol Phys. 2006; 66(2): 560-567, doi: 10.1016/j. ijrobp.2006.06.004, indexed in Pubmed: 16965998.

5. Torres Royo L, Antelo Redondo G, Árquez Pianetta M, et al. Low-Dose radiation therapy for benign pathologies. Rep Pract Oncol Radiother. 2020; 25(2): 250-254, doi: 10.1016/j.rpor.2020.02.004, indexed in Pubmed: 32140081.

6. Moldeveanu B, Otmishi P, Jani P, et al. Inflammatory mechanisms in the lung. Journal of Inflammation Reaserch. 2009: 1-11.

7. Schröder S, Kriesen S, Paape D, et al. Modulation of Inflammatory Reactions by Low-Dose lonizing Radiation: Cytokine Release of Murine Endothelial Cells Is Dependent on Culture Conditions. J Immunol Res. 2018; 2018: 2856518, doi: 10.1155/2018/2856518, indexed in Pubmed: 29967799.

8. Ameri A, Rahnama N, Bozorgmehr R, et al. Low-Dose Whole-Lung Irradiation for COVID-19 Pneumonia: Short Course Results. Int J Radiat Oncol Biol Phys. 2020; 108(5): 1134-1139, doi: 10.1016/j. ijrobp.2020.07.026, indexed in Pubmed: 32707264.

9. Kirkby C, Mackenzie M. Is low dose radiation therapy a potential treatment for COVID-19 pneumonia? Radiother Oncol. 2020; 147: 221, doi: 10.1016/j.radonc.2020.04.004, indexed in Pubmed: 32342871.

10. Taghizadeh-Hesary F, Akbari H. The powerful immune system against powerful COVID-19: A hypothesis. Med Hypotheses. 2020; 140: 109762, doi: 10.1016/j.mehy.2020.109762, indexed in Pubmed: 32388390.

11. ECLIPSE Photon and Electron Algorithms Reference Guide, P1020505-003-C, Varian Medical Systems. Acesso em: July 2018. 